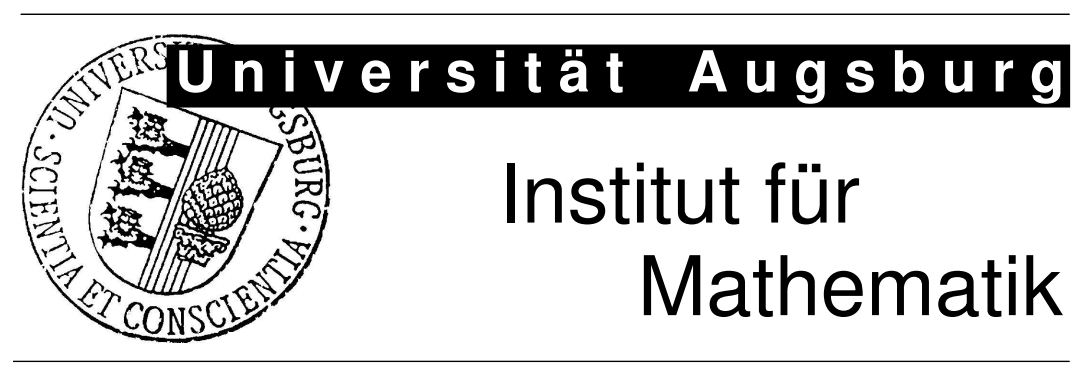

Malte A. Peter, Michael H. Meylan

A General Spectral Approach to the Time-Domain Evolution of Linear Water Waves Impacting on a Vertical Elastic Plate 


\section{Impressum:}

\section{Herausgeber:}

Institut für Mathematik

Universität Augsburg

86135 Augsburg

http://www.math.uni-augsburg.de/pages/de/forschung/preprints.shtml

\section{ViSdP:}

Malte A. Peter

Institut für Mathematik

Universität Augsburg

86135 Augsburg

Preprint: Sämtliche Rechte verbleiben den Autoren (C) 2009 


\title{
A GENERAL SPECTRAL APPROACH TO THE TIME-DOMAIN EVOLUTION OF LINEAR WATER WAVES IMPACTING ON A VERTICAL ELASTIC PLATE*
}

\author{
MALTE A. PETER ${ }^{\dagger}$ AND MICHAEL H. MEYLAN ${ }^{\ddagger}$
}

\begin{abstract}
We present a solution in the time-domain to the two-dimensional linear water-wave problem, in which a semi-infinite fluid region is bounded on one side by a vertical elastic plate. The problem is solved using a generalized eigenfunction expansion from the solutions for single frequencies and we begin with a novel solution of the single-frequency problem. By formulating the problem using the acceleration potential, we find an inner-product space, in which the evolution operator with continuous spectrum is self-adjoint. This inner-product space is required for the generalized eigenfunction solution, which allows to prescribe arbitrary initial water surface and plate displacements and velocities. Furthermore, using the generalized eigenfunction expansion, the solution is approximated by deforming the contour of integration and using the contributions from the singularities of the analytic continuation. Numerical experiments show that the long-time behavior in certain situations can be well approximated by this method.
\end{abstract}

Key words. Water waves, Spectral theory, Generalized eigenfunction expansion, Scattering, Complex scattering frequency.

AMS subject classifications. 76B15, 76M22, 74F10, 35P25, 47A40.

1. Introduction. Hydroelasticity is the study of the interaction of fluids and elastic bodies. The present paper concerns a particular hydroelastic problem, in which a semi-infinite fluid interacts with a vertical elastic plate. The problem is motivated by both trying to understand the ways, in which waves interact with the vertical plate, and how the vibration of the vertical plate is affected by the presence of the fluid. The former problem is motivated by wave-impact problems, which occur in tanks of fluid, and the latter problem is concerned with the understanding of tank-wall vibration. It turns out that the two problems are closely connected mathematically and we solve both problems in a unified approach in what follows.

The linear problem of a vertical elastic plate, which forms the right-hand boundary of a semi-infinite two-dimensional fluid domain, has not been well studied and we present here a novel method even to solve for the single-frequency solutions. The corresponding problem of a floating elastic plate has received enormous research consideration on the other hand, mainly motivated by the study of ocean-wave interaction with sea ice [24, 23] or so-called Very Large Floating Structures [26]. It is worth pointing out that violent wave impact (i.e. very steep or breaking waves) on walls has been studied substantially, both theoretically and experimentally, cf. [11, 20, 12, 2] for example. In this context, other effects become important such as nonlinearity of the waves and air entrapment, which are not accounted for within the linearized theory employed here. However, an elastic plate, which is displaced by a violent impact, may very well oscillate and decay according to the linear theory we present here.

We solve the problem using a generalized eigenfunction expansion, which is a generalization of the standard eigenfunction method on bounded domains to domains in

*A PRELIMINARY SHORT SUMMARY OF THE RESULTS HAS APPEARED IN [21].

${ }^{\dagger}$ Department of Mathematics, University of Auckland, Private Bag 92019, Auckland 1142, New Zealand. Current address: Institute of Mathematics, University of Augsburg, 86135 Augsburg, Germany and Augsburg Centre for Innovative Technologies, University of Augsburg, Germany (malte. peter@math. uni-augsburg.de).

${ }^{\ddagger}$ Department of Mathematics, University of Auckland, Private Bag 92019, Auckland 1142, New Zealand (meylan@math.auckland.ac.nz). 
which energy can radiate to infinity. The existence of an infinite boundary complicates the standard problem in two ways. Firstly, the spectrum becomes continuous so that the normalization of the eigenfunctions becomes non-trivial and, secondly, the existence of a boundary at infinity also makes the boundary conditions, which must be imposed, non-trivial. However, the eigenfunctions associated with the continuous spectrum are exactly the single-frequency solutions, which are the object of principle interest and for which many methods of solution have been developed. The generalized eigenfunction method began with the work of [22, 10] for Schrödinger's equations and was extended for water waves by $[4,1,6,7]$. All of these solutions were theoretical and the use of this method for numerical calculations does not seem to have been well investigated. Numerical methods were only recently developed for the case of floating plates by [9], for rigid bottom-mounted cylinders in three dimensions in [18] and for general fixed bodies in two dimensions by [17].

Besides supplying an easily computable solution for the water-wave scattering by an elastic wall, the other main aim of the paper is to further develop the generalizedeigenfunction-expansion method. It is to be expected that the method could be extended to almost any scattering problem, which involves a domain with boundaries at infinity. However, each time a new scattering term appears, a very careful treatment is required. In the present work, this novel complication arises from the semi-infinite domain with vertical boundary given by a plate and by the plate extending beyond the fluid. Furthermore, the current new presentation of the method is clearer than in previous works and, in particular, does not involve positive and negative frequencies, which avoids many of the technicalities.

It is well known that, in certain situations, systems display resonance behavior. In our case, this is associated with a plate of much greater density than the fluid and which is therefore only lightly damped. In this case, while there is no point in the discrete spectrum, there is a singularity in the analytic extension of the solution (resolvent) close to the real axis, a so-called complex scattering frequency. The approximation of the solution using this singularity via the Laplace transform is known as the singularity-expansion method. This method may be viewed as a generalization of Lax-Phillips scattering [13] to problems, in which the incoming and outgoing subspaces are not orthogonal (as is the case for the present problem under study). The approach we take here is somewhat different, and we use the generalized eigenfunction solution rather than the Laplace-transform solution as our starting point. It should be noted that, because we can determine the single-frequency solutions so easily, the generalized eigenfunction solution is much simpler than the equivalent solution using a Laplace-transform method such as the singularity-expansion method [5, 8] (or any other related methods such as the memory-effect method [16]).

Our aim here is to solve the time-domain problem of a vertical elastic plate forming the right-hand boundary of a two-dimensional semi-infinite fluid domain. We expand the solution in terms of the single-frequency solutions using a generalized eigenfunction expansion. The generalized eigenfunction expansion allows us to express the solution in the time-domain for initial conditions for both the fluid surface and the plate. We then consider an approximation method based on singularities of the analytic extension combined with contour integration of the Fourier-type integrals, which appear in the generalized eigenfunction expansion. From this approximation method, modes are calculated, which may be thought of as corresponding to the modes of vibration of a plate vibrating against a semi-infinite fluid. Care has to be taken in the interpretation of these modes, because the mathematical structure of the problem 


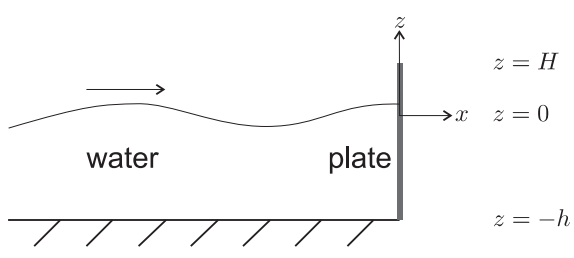

FIG. 2.1. Sketch of the problem setting.

is different from the traditional vibrational problems for a self-adjoint problem. For example, these modes are valid only close to the plate.

The paper is organized as follows: We begin with the precise formulation of the problem (§2) and the solution of the single-frequency problem (§3). Section 4 is concerned with the solution of the time-domain problem using the generalized eigenfunction expansion method. The approximation method using complex scattering frequencies is developed in $\S 5$. Numerical experiments are presented in $\S 6$ and a summary and discussion of the results are given in $\S 7$. The paper has three appendices discussing the roots of the dispersion relation for complex frequencies $(\S \mathrm{A})$, some details about the eigenfunctions of the plate $(\S \mathrm{B})$ as well as some properties of the evolution operator $\mathcal{A}(\S \mathrm{C})$.

2. Statement of the problem and mathematical formulation. The problem consists of a semi-infinite fluid domain $\Omega=\left\{(x, z) \in \mathbb{R}^{2} \mid-\infty<x<0,-h<\right.$ $z<0\}$, where $z=0$ represents the free water surface and where there is a vertical elastic plate at $x=0$, the boundaries of which may extend beyond the domain. In particular, the height of the plate extends from $-h$ to $H$ with $H \geq 0$. A sketch of the setting can be found in figure 2.1. We assume the fluid flow is irrotational and that it can be described using linear inviscid theory. The plate is governed by the BernoulliEuler plate equation. In all of what follows, we work with standard dimensionless coordinates, where the spatial variables have been scaled by a length parameter $L$ and the time variable by $\sqrt{L / g}$, where $g$ is the gravitational acceleration.

2.1. Governing equations. The mathematical description of the problem is as follows. We use the acceleration potential $\Psi$ to describe the fluid motion, rather than the velocity potential as is standard. This makes the equations in the time-domain simpler and was used in [9] for the generalized eigenfunction solution for a floating plate. For all times $t>0$, the potential $\Psi(x, z, t)$ satisfies

$$
\begin{aligned}
-\Delta \Psi & =0, & & (x, z) \in \Omega, \\
\partial_{n} \Psi & =0, & & -\infty<x<0, z=-h,
\end{aligned}
$$

where $\partial_{n}$ is the outward normal derivative. The kinematic condition at the free-surface is

$$
\partial_{t}^{2} Z=\partial_{n} \Psi, \quad-\infty<x<0, z=0,
$$

where $Z(x, t)$ is the displacement of the water surface. The dynamic condition is

$$
Z+\Psi=0, \quad-\infty<x<0, z=0,
$$

(note that this condition would read $g Z+\Psi=0$ in standard dimensional coordinates.) 
The plate is governed by the Bernoulli-Euler plate equation. There is a force on the wetted surface of the plate from the water (given by $p=-\rho \Psi$, where $\rho$ is the water density) while there is no force above the free water surface. The dimensionless governing equation for the plate displacement $W(z, t)$ thus reads

$$
\gamma \partial_{t}^{2} W+\beta \partial_{z}^{4} W= \begin{cases}0, & 0 \leq z<H \\ -\Psi, & -h<z<0\end{cases}
$$

subject to edge conditions, where the positive parameters $\beta$ and $\gamma$ are related to the mass and the stiffness of the plate, resp. In what follows, we consider the plate clamped at the bottom, i.e.

$$
W=0, \partial_{z} W=0, \quad z=-h .
$$

At the top, the plate is either clamped, i.e.

$$
W=0, \partial_{z} W=0, \quad z=H,
$$

or free to move, i.e.

$$
\partial_{z}^{2} W=0, \partial_{z}^{3} W=0, \quad z=H .
$$

Other boundary conditions could be considered within the framework of this work. For convenience, we write $\chi$ for the characteristic function of the wetted plate surface, i.e. $\chi(z)=1$ for $z<0$ and $\chi(z)=0$ for $z \geq 0$.

The system of equations is completed by initial conditions for the water surface and plate displacements and their velocities:

$$
\begin{array}{clrl}
Z(x, 0) & =Z_{0}(x), \partial_{t} Z(x, 0)=Z_{1}(x), & & -\infty<x<0, \\
W(z, 0) & =W_{0}(z), \partial_{t} W(z, 0)=W_{1}(z), & & -h<z<H .
\end{array}
$$

2.2. Formulations in terms of plate modes. We assume we have an infinite set of orthonormal modes $w_{n}(z), n=0,1, \ldots$, which satisfy the equation $\partial_{z}^{4} w_{n}=$ $\lambda_{n}^{4} w_{n}$ as well as the appropriate boundary conditions at the ends of the plates (which may extend beyond the boundary of the fluid domain). The discussion of these modes is given in Appendix B. This allows us to express the displacement of the plate as

$$
W(z, t)=\sum_{n=0}^{\infty} \alpha_{n}(t) w_{n}(z) .
$$

At $x=0$, the plate displacement and the acceleration potential are related through the kinematic and dynamic conditions. The equations coupling the water acceleration potential and the plate displacement are therefore

$$
-\chi \Psi=\gamma \sum_{n=0}^{\infty} \partial_{t}^{2} \alpha_{n} w_{n}+\beta \sum_{n=0}^{\infty} \lambda_{n}^{4} \alpha_{n} w_{n}
$$

and, along the wetted surface of the plate,

$$
\sum_{n=0}^{\infty} \partial_{t}^{2} \alpha_{n} w_{n}=\partial_{n} \Psi
$$

For $z<0$, combining the last two equations gives

$$
-\Psi-\gamma \partial_{n} \Psi=\beta \sum_{n=0}^{\infty} \lambda_{n}^{4} \alpha_{n} w_{n} .
$$


3. Single-frequency solution. In this section, the problem is solved under the assumption that the acceleration potential and the surface displacement are time harmonic with given radian frequency $\omega$, i.e.

$$
\Psi(x, z, t)=\operatorname{Re}\left\{\psi(x, z) \mathrm{e}^{-\mathrm{i} \omega t}\right\}, \quad Z(x, t)=\operatorname{Re}\left\{\zeta(x) \mathrm{e}^{-\mathrm{i} \omega t}\right\}
$$

with a complex potential $\psi$ and complex displacement $\zeta$.

At the free surface, condition (2.1c) simplifies to

$$
-\omega^{2} \zeta=\partial_{n} \psi, \quad z=0
$$

This can be combined with equation (2.1d) to give the single free-surface condition

$$
\alpha \psi=\partial_{z} \psi, \quad z=0,
$$

where $\alpha=\omega^{2}$ (in dimensional coordinates, this would read $\omega^{2} / g$ ). The positive wavenumber $k$ is related to $\alpha$ by the dispersion relation

$$
\alpha=k \tanh k h .
$$

See [14] e.g., the acceleration potential takes the form

$$
\psi(x, z)=A^{\mathrm{I}} f_{0}(z) \mathrm{e}^{-k_{0} x}+\sum_{m=0}^{\infty} c_{m} f_{m}(z) \mathrm{e}^{k_{m} x}
$$

where the first term is due to the ambient incident potential of amplitude $A^{\mathrm{I}}$ and the coefficients $c_{m}$ are of the scattered wavefield only. The functions

$$
f_{m}(z)=\frac{\cos k_{m}(z+h)}{\cos k_{m} h}
$$

are the vertical eigenfunctions. The numbers $k_{m}, m \geq 1$, are given as positive real roots of the dispersion relation

$$
\alpha+k_{m} \tan k_{m} h=0
$$

and $k_{0}=-\mathrm{i} k$. Note that $k_{0}$ is a (purely imaginary) root of (3.7). Moreover, we have

$$
\int_{-h}^{0} f_{m}(z) f_{n}(z) \mathrm{d} z=\delta_{m n} N_{m}
$$

where $N_{m}$ is given by

$$
N_{m}=\frac{1}{2} \frac{\cos k_{m} h \sin k_{m} h+k_{m} h}{k_{m} \cos ^{2} k_{m} h} .
$$

Using (3.5) at $x=0$ in (2.9) gives

$$
\chi(z)\left(-A^{\mathrm{I}} f_{0}(z)-\sum_{m=0}^{\infty} c_{m} f_{m}(z)\right)=\sum_{n=0}^{\infty}\left(-\gamma \omega^{2}+\beta \lambda_{n}^{4}\right) \alpha_{n} w_{n}(z) .
$$

Multiplying by $w_{k}$ and integrating from $-h$ to $H$ with respect to the vertical variable, we obtain

$$
-A^{\mathrm{I}} \int_{-h}^{0} w_{k}(z) f_{0}(z) \mathrm{d} z-\sum_{m=0}^{\infty} c_{m} \int_{-h}^{0} w_{k}(z) f_{m}(z) \mathrm{d} z=\left(-\gamma \omega^{2}+\beta \lambda_{k}^{4}\right) \alpha_{k} .
$$


The second equation can be obtained from (2.10). For time harmonic motion, it reads

$$
-\omega^{2} \sum_{n=0}^{\infty} \alpha_{n} w_{n}(z)=-k_{0} A^{\mathrm{I}} f_{0}(z)+\sum_{m=0}^{\infty} k_{m} c_{m} f_{m}(z) .
$$

Multiplying by $f_{l}$ and integrating as before gives

$$
-\omega^{2} \sum_{n=0}^{\infty} \alpha_{n} \int_{-h}^{0} w_{n}(z) f_{l}(z) \mathrm{d} z=-k_{0} N_{0} A^{\mathrm{I}} \delta_{l 0}+k_{l} N_{l} c_{l} .
$$

We have thus derived a system of equations for the unknown coefficients $\alpha_{n}$ and $c_{m}$ :

$$
\begin{aligned}
\alpha_{n}+ & \frac{1}{\beta \lambda_{n}^{4}-\gamma \omega^{2}} \sum_{m=0}^{\infty} c_{m} \int_{-h}^{0} w_{n}(z) f_{m}(z) \mathrm{d} z=-\frac{A^{\mathrm{I}}}{\beta \lambda_{n}^{4}-\gamma \omega^{2}} \int_{-h}^{0} w_{n}(z) f_{0}(z) \mathrm{d} z, & n \in \mathbb{N}, \\
& \frac{\omega^{2}}{k_{m} N_{m}} \sum_{n=0}^{\infty} \alpha_{n} \int_{-h}^{0} w_{n}(z) f_{m}(z) \mathrm{d} z+c_{m}=A^{\mathrm{I}} \delta_{m 0}, & m \in \mathbb{N} .
\end{aligned}
$$

For future reference, we note that this system can be written in the form

$$
(I+A(k)) c(k)=b(k),
$$

where $c(k)=\left(\alpha_{n}(k), c_{m}(k)\right)$ and $I$ is the identity operator, and where we have explicitly expressed the dependence on the wavenumber $k$, which is given a priori in the single-frequency case (as it is uniquely determined from (3.4) for given $\omega$ ).

The system (3.14) can be solved very efficiently numerically by truncation. The functions $w_{n}$ and $f_{m}$ are given in closed form (also see Appendix B) and the integrals in (3.14) can be calculated analytically. Moreover, we note that the dimension of the linear system of equations (3.14) can be reduced by eliminating the coefficients $c_{m}$ or $\alpha_{n}$. This can be achieved easily by solving (3.13) for $c_{l}$ and substituting into (3.11) or by using (3.11) in (3.13), respectively.

4. Time-dependent problem. We want to use the time-harmonic solutions for fixed frequencies to construct the solution for arbitrary initial conditions. From the nature of the governing equations, four functions need to be prescribed: the initial displacement $Z_{0}(x)$ and velocity $Z_{1}(x)$ of the water surface and the initial displacement $W_{0}(z)$ and velocity $W_{1}(z)$ of the plate.

From the linearity of the problem, it is clear that a superposition of the singlefrequency solutions will be a solution of the time-dependent problem. However, it is also true that any solution in time domain can be written as a linear combination of single-frequency solutions with certain frequency- (or wavenumber-) dependent weights. It is not clear, however, how these weighting functions must be chosen in order to meet the initial conditions. The remainder of this section is devoted to the this problem, which we solve using the generalized eigenfunction method based on a spectral formulation.

4.1. Spectral Formulation and general solution. We want to rewrite the governing equations in the form of an abstract wave equation. This can be done in terms of the surface and plate displacements in the two-component system

$$
\partial_{t}^{2}\left(\begin{array}{c}
Z \\
W
\end{array}\right)+\left(\begin{array}{ll}
\mathcal{A}_{11} & \mathcal{A}_{12} \\
\mathcal{A}_{21} & \mathcal{A}_{22}
\end{array}\right)\left(\begin{array}{c}
Z \\
W
\end{array}\right)=0
$$


The operators $\mathcal{A}_{i j}$ are Dirichlet-to-Neumann-type operators mapping in the following way: $\mathcal{A}_{11}: Z \mapsto-\left.\Psi_{n}\right|_{\Gamma^{\mathrm{f}}}, \mathcal{A}_{12}: W \mapsto-\left.\Psi_{n}\right|_{\Gamma^{\mathrm{f}}}, \mathcal{A}_{21}: Z \mapsto-\left.\partial_{t}^{2} W\right|_{\Gamma^{\mathrm{p}}}$ and $\mathcal{A}_{22}: W \mapsto$ $-\left.\partial_{t}^{2} W\right|_{\Gamma^{\mathrm{p}}}$ In all cases, $\Psi$ is the solution of the boundary-value problem

$$
\begin{aligned}
-\Delta \Psi & =0, & & -h<z<0, \\
\partial_{z} \Psi & =0, & & z=-h,
\end{aligned}
$$

and the remaining boundary conditions are given by:

$$
\begin{array}{lll} 
& \text { free surface } & \text { wetted plate } \\
\mathcal{A}_{j 1} & \Psi=-Z & \Psi=-\gamma \Psi_{n} \\
\mathcal{A}_{j 2} & \Psi=0 & \Psi=-\gamma \Psi_{n}-\beta \partial_{z}^{4} W
\end{array}
$$

For $A_{2 j}$, the potential at the wetted plate further needs to be mapped to the acceleration of the full plate. For $\mathcal{A}_{21}$, we have to use

$$
\gamma \partial_{t}^{2} W=-\chi \Psi
$$

while for $\mathcal{A}_{22}$, we require

$$
\gamma \partial_{t}^{2} W=-\beta \partial_{z}^{4} w-\chi \Psi,
$$

each subject to the edge conditions prescribed for the plate.

The operator

$$
\mathcal{A}=\left(\begin{array}{ll}
\mathcal{A}_{11} & \mathcal{A}_{12} \\
\mathcal{A}_{21} & \mathcal{A}_{22}
\end{array}\right)
$$

is self-adjoint in the domain of $\mathcal{A}$ equipped with the inner product

$$
\left\langle\left(\begin{array}{c}
Z \\
W
\end{array}\right),\left(\begin{array}{c}
Z^{\prime} \\
W^{\prime}
\end{array}\right)\right\rangle_{\mathcal{A}}=\left\langle Z, Z^{\prime}\right\rangle_{\Gamma^{\mathrm{f}}}+\beta\left\langle\partial_{z}^{2} W, \partial_{z}^{2} W^{\prime}\right\rangle_{\Gamma^{\mathrm{p}}},
$$

where $\langle\cdot, \cdot\rangle_{\Gamma}$ denotes the standard inner product of $L^{2}(\Gamma)$, cf. Appendix C.1 for the symmetry of $\mathcal{A}$. It has a continuous spectrum: $[0, \infty)$. The corresponding generalized eigenfunctions are precisely the single-frequency solutions for a unit-amplitude incident wave, $(\hat{\zeta}(x, k), \hat{w}(z, k))$.

Thus, any solution of (4.1) can be written in the form

$$
\begin{aligned}
\left(\begin{array}{c}
Z(x, t) \\
W(z, t)
\end{array}\right)=\sqrt{\frac{2}{\pi}} \int_{0}^{\infty} \mathfrak{f}_{1}(k)\left(\begin{array}{c}
\hat{\zeta}(x, k) \\
\hat{w}(z, k)
\end{array}\right) \cos \omega(k) t \\
+\mathfrak{f}_{2}(k)\left(\begin{array}{c}
\hat{\zeta}(x, k) \\
\hat{w}(z, k)
\end{array}\right) \frac{\sin \omega(k) t}{\omega(k)} \mathrm{d} k
\end{aligned}
$$

with wavenumber-dependent weighting functions $\mathfrak{f}_{1}(k)$ and $\mathfrak{f}_{2}(k)$. From Appendix C.2, we have

$$
\left\langle\left(\begin{array}{c}
\hat{\zeta}(\cdot, k) \\
\hat{w}(\cdot, k)
\end{array}\right),\left(\begin{array}{c}
\hat{\zeta}(\cdot, \kappa) \\
\hat{w}(\cdot, \kappa)
\end{array}\right)\right\rangle_{\mathcal{A}}=2 \pi \delta(k-\kappa)
$$

Thus, for the initial displacement of the free surface $Z_{0}$ and of the plate $W_{0}$, we obtain

$$
\left(\begin{array}{c}
Z_{0}(x) \\
W_{0}(z)
\end{array}\right)=\sqrt{\frac{2}{\pi}} \int_{0}^{\infty} \mathfrak{f}_{1}(k)\left(\begin{array}{c}
\hat{\zeta}(x, k) \\
\hat{w}(z, k)
\end{array}\right) \mathrm{d} k
$$


and we find that

$$
\begin{aligned}
\left\langle Z_{0}, \hat{\zeta}(\cdot, \kappa)\right\rangle_{\Gamma^{\mathrm{f}}}+\beta\left\langle\partial_{z}^{2} W_{0}, \partial_{z}^{2} \hat{w}(\cdot, \kappa)\right\rangle_{\Gamma^{\mathrm{p}}} & \\
& =\left\langle\left(\begin{array}{c}
Z_{0} \\
W_{0}
\end{array}\right),\left(\begin{array}{c}
\hat{\zeta}(\cdot, \kappa) \\
\hat{w}(\cdot, \kappa)
\end{array}\right)\right\rangle_{\mathcal{A}}=2 \sqrt{2 \pi} \mathfrak{f}_{1}(\kappa) .
\end{aligned}
$$

Hence,

$$
\int_{\Gamma^{\mathrm{f}}} Z_{0}(x) \overline{\hat{\zeta}}(x, \kappa) \mathrm{d} x+\beta \int_{\Gamma^{\mathrm{P}}} \partial_{z}^{2} W_{0}(z) \partial_{z}^{2} \overline{\hat{w}}(z, \kappa) \mathrm{d} z=2 \sqrt{2 \pi} \mathfrak{f}_{1}(\kappa),
$$

where the overbar denotes complex conjugation, and, similarly,

$$
\int_{\Gamma^{\mathrm{f}}} Z_{1}(x) \overline{\hat{\zeta}}(x, \kappa) \mathrm{d} x+\beta \int_{\Gamma^{\mathrm{p}}} \partial_{z}^{2} W_{1}(z) \partial_{z}^{2} \overline{\hat{w}}(z, \kappa) \mathrm{d} z=2 \sqrt{2 \pi} \mathfrak{f}_{2}(\kappa)
$$

are obtained, which determine the functions $\mathfrak{f}_{1}$ and $\mathfrak{f}_{2}$ in the representation (4.5).

In what follows, we consider in detail problems with vanishing initial velocities. In such cases, $\mathfrak{f}_{2}=0$ and (4.5) simplifies to

$$
\left(\begin{array}{c}
Z(x, t) \\
W(z, t)
\end{array}\right)=\sqrt{\frac{2}{\pi}} \int_{0}^{\infty} \mathfrak{f}_{1}(k)\left(\begin{array}{c}
\hat{\zeta}(x, k) \\
\hat{w}(z, k)
\end{array}\right) \cos \omega(k) t \mathrm{~d} k .
$$

As the product of $\mathfrak{f}_{1}$ and the single-frequency solution must be real (in particular, $Z$ and $W$ are real and so is $\cos \omega t$ ), this can be rewritten equivalently as

$$
\left(\begin{array}{c}
Z(x, t) \\
W(z, t)
\end{array}\right)=\frac{2}{\sqrt{2 \pi}} \operatorname{Re}\left[\int_{0}^{\infty} \mathfrak{f}_{1}(k)\left(\begin{array}{c}
\hat{\zeta}(x, k) \\
\hat{w}(z, k)
\end{array}\right) \mathrm{e}^{-\mathrm{i} \omega(k) t} \mathrm{~d} k\right] .
$$

We use this form in the derivation of the approximation method in $\S 5$ as it is more convenient for the manipulations carried out there. An expression analogous to (4.11) can be derived for vanishing initial displacements, i.e. $\mathfrak{f}_{1}=0$.

It is worth noting that it is generally equivalent to work with complex exponentials as in (4.11) and (4.16) or with trigonometric functions as in (4.5). See [3] e.g., writing $u(t)=(Z(t), W(t))^{T}$, the solution of problem (4.1) can be expressed in the two equivalent forms

$$
\begin{aligned}
u(t) & =\mathcal{A}^{-\frac{1}{2}} \operatorname{Re}\left[\mathrm{e}^{-\mathrm{i} \mathcal{A}^{\frac{1}{2}} t}\left(\mathcal{A}^{\frac{1}{2}} u_{0}+\mathrm{i} u_{1}\right)\right] \\
& =\cos \left(\mathcal{A}^{\frac{1}{2}} t\right) u_{0}+\mathcal{A}^{-\frac{1}{2}} \sin \left(\mathcal{A}^{-\frac{1}{2}} t\right) u_{1} .
\end{aligned}
$$

Both formulations have advantages and disadvantages. In particular, while the trigonometric approach leads to simpler formulas for the solution of the initial-boundary value problem (cf. (4.9)), the complex exponential is more convenient in the derivation of the approximation theory presented in $\S 5$.

4.2. Canonical initial conditions. We discuss in detail the cases where there is either only a non-zero initial water surface displacement or only a non-zero initial plate displacement. Similar expressions are derived if only one of the initial velocities is nonzero. For vanishing initial velocities, the water surface and plate displacements are given by (4.10) or (4.11). We also derive a very simple expression for a pulse initially travelling toward the plate from far away, which is an important initial condition for numerical experiments in engineering applications. 
4.2.1. Initial surface displacement. If we have non-zero initial surface displacement $Z_{0}$ while $Z_{1}, W_{0}, W_{1}=0, \mathfrak{f}_{2}$ vanishes and (4.9a) simplifies to

$$
\mathfrak{f}_{1}(\kappa)=\frac{1}{2 \sqrt{2 \pi}} \int_{\Gamma^{\mathrm{f}}} Z_{0}(x) \overline{\hat{\zeta}}(x, \kappa) \mathrm{d} x .
$$

A simplification can be made if the initial surface displacement vanishes near the plate. In this case, the evanescent waves in $\overline{\hat{\zeta}}$ have already decayed virtually completely at the location of the pulse. Thus,

$$
\mathfrak{f}_{1}(\kappa)=\frac{1}{2 \sqrt{2 \pi}} \int_{\Gamma^{\mathrm{f}}} Z_{0}(x)\left(\mathrm{e}^{-\mathrm{i} \kappa x}+\bar{c}_{0}(\kappa) \mathrm{e}^{\mathrm{i} \kappa x}\right) \mathrm{d} x .
$$

It can be shown further that (4.10) with (4.15) reduces to

$$
\left(\begin{array}{c}
Z(x, t) \\
W(z, t)
\end{array}\right)=\frac{2}{\sqrt{2 \pi}} \operatorname{Re}\left[\int_{0}^{\infty} \mathfrak{f}(k)\left(\begin{array}{c}
\hat{\zeta}(x, k) \\
\hat{w}(z, k)
\end{array}\right) \cos \omega(k) t \mathrm{~d} k\right]
$$

in this case, where

$$
\mathfrak{f}(k)=\frac{1}{\sqrt{2 \pi}} \int_{\Gamma^{\mathrm{f}}} Z_{0}(x) \mathrm{e}^{-\mathrm{i} k x} \mathrm{~d} x .
$$

4.2.2. Initial plate displacement. If we have non-zero initial plate displacement $W_{0}$ while $Z_{0}, Z_{1}, W_{1}=0,(4.9 \mathrm{a})$ simplifies to

$$
\mathfrak{f}_{1}(\kappa)=\frac{\beta}{2 \sqrt{2 \pi}} \int_{\Gamma^{\mathrm{p}}} \partial_{z}^{2} W_{0}(z) \partial_{z}^{2} \overline{\hat{w}}(z, \kappa) \mathrm{d} z,
$$

and $\mathfrak{f}_{2}=0$. Representing $W_{0}$ in terms of the plate eigenfunctions, $W_{0}=\sum_{n=0}^{\infty} \alpha_{n}^{0} w_{n}$ say, this further simplifies to

$$
\mathfrak{f}_{1}(\kappa)=\frac{\beta}{2 \sqrt{2 \pi}} \sum_{n=0}^{\infty} \alpha_{n}^{0} \lambda_{n}^{4} \overline{\hat{\alpha}}_{n}(\kappa) .
$$

4.2.3. Incident pulse from far away. The case of an incident pulse hitting the plate can be treated as follows. If the plate is at rest at $t=0$ and the initial water-surface displacement vanishes near the plate, (4.14) reduces to (4.15). In the same way, (4.9b) simplifies to

$$
\mathfrak{f}_{2}(\kappa)=\frac{1}{2 \sqrt{2 \pi}} \int_{-\infty}^{0} Z_{1}(x)\left(\mathrm{e}^{-\mathrm{i} \kappa x}+\bar{c}_{0}(\kappa) \mathrm{e}^{\mathrm{i} \kappa x}\right) \mathrm{d} x .
$$

It is not obvious from this representation what $Z_{1}$ is in order for the pulse to travel to the right. This can be achieved easily, however, noting that taking a time derivative is equivalent to multiplying with $\pm \mathrm{i} \omega$ in the Fourier domain. Choosing the sign such that the pulse travels to the right (i.e. toward the plate) gives

$$
\mathfrak{f}_{2}(\kappa)=\frac{\mathrm{i} \omega}{2 \sqrt{2 \pi}} \int_{-\infty}^{0} Z_{0}(x)\left(-\mathrm{e}^{-\mathrm{i} \kappa x}+\bar{c}_{0}(\kappa) \mathrm{e}^{\mathrm{i} \kappa x}\right) \mathrm{d} x .
$$

Similarly to (4.16), (4.10) reduces to

$$
\left(\begin{array}{c}
Z(x, t) \\
W(z, t)
\end{array}\right)=\frac{2}{\sqrt{2 \pi}} \operatorname{Re}\left[\int_{0}^{\infty} \mathfrak{f}(k)\left(\begin{array}{c}
\hat{\zeta}(x, k) \\
\hat{w}(z, k)
\end{array}\right) \mathrm{e}^{-\mathrm{i} \omega(k) t} \mathrm{~d} k\right]
$$

in this case, where $\mathfrak{f}$ is given by (4.17). 
5. Approximation of near-resonant behavior. Near-resonant behavior occurs when a complex scattering frequency is close to the real axis. Thus, the complex scattering frequencies are discussed first. For the situation of a complex scattering frequency occurring near the real axis, an approximation of the solution for large times is given.

5.1. Complex scattering frequencies. The system of equations (3.14) can be written in the form (3.15), i.e.

$$
(I+A(k)) c(k)=b(k) .
$$

Note that we have explicitly expressed the dependence on the parameter $k$. This equation is typical of scattering problems, in that we have an operator plus the identity. This is because the scattering process is a perturbation of the incident wave, and in the absence of scattering we have simply the identity. Exactly such an equation appears in [5]. The explicit solution to the scattering problem is of course given by $c(k)=(I+A(k))^{-1} b(k)$.

The key to understanding this equation is to allow the parameter $k$ to become complex. We then find that the operator possesses zero eigenvalues in the lower complex plane (which are equivalent to the eigenvalues of the operator $A(k)$ with eigenvalue -1$)$. It is obvious that, where these zeros occur, $c(k)$ is singular, i.e. the system has an infinite response (note that this is not an infinite response for a real frequency unless there is a trapped mode). These zeros are called complex scattering wavenumbers and, more commonly, the corresponding frequencies $\omega$ are known as complex scattering frequencies. For very special situations for the general water-wavescattering problem, a purely real zero can occur, which is known as a trapped mode. We do not expect to find any trapped modes in the situation under consideration here and they have never been found. However, as will be seen later on (cf. §6), there are situations of near-trapping or near-resonance, where a scattering frequency is close to the real axis. The aim of the present work is to examine what happens when the scattering frequencies become close to the real axis. This allows us to make predictions of the response in the time and frequency domains.

5.2. Approximation for large times. Suppose there is a scattering frequency at a complex wavenumber $k_{\mathrm{r}}$. The scattering wavenumber $k_{\mathrm{r}}$ is associated with an eigenvector with zero eigenvalue. That is, there is an eigenvector $u_{k_{\mathrm{r}}}$ with the property that $\left(I+A\left(k_{\mathrm{r}}\right)\right) u_{k_{\mathrm{r}}}=0$. Then we know that near the pole the inverse of $I+A\left(k_{\mathrm{r}}\right)$ can be written as

$$
(I+A(k))^{-1} \approx \frac{R\left(k_{\mathrm{r}}\right)}{k-k_{\mathrm{r}}},
$$

where $R\left(k_{\mathrm{r}}\right)$ is the residue which is connected with a projection onto the eigenspace associated with $k_{\mathrm{r}}$. We can derive the expression for $R\left(k_{\mathrm{r}}\right)$ using a result due to [25] for the case of a simple zero (the eigenspace has dimension one) at $k_{\mathrm{r}}$. It is also possible to treat roots of higher multiplicity. Since we do not expect such roots to occur in this problem we do not follow this direction here. It can be shown that

$$
R\left(k_{\mathrm{r}}\right)=\frac{\left\langle u_{k_{\mathrm{r}}}, u_{\bar{k}_{\mathrm{r}}}\right\rangle}{\left\langle A^{\prime}\left(k_{\mathrm{r}}\right) u_{k_{\mathrm{r}}}, u_{\bar{k}_{\mathrm{r}}}\right\rangle}
$$


[25], where $u_{\bar{k}_{\mathrm{r}}}$ is the eigenvector of the adjoint operator with eigenvalue zero and $A^{\prime}\left(k_{\mathrm{r}}\right)$ is the derivative of $A$ at $k_{\mathrm{r}}$ given by

$$
A^{\prime}\left(k_{\mathrm{r}}\right)=\left.\frac{\mathrm{d}}{\mathrm{d} k} A(k)\right|_{k=k_{\mathrm{r}}} .
$$

For future reference, we denote by $k_{\mathrm{r}}$ the complex value of $k$ closest to the real axis, for which $(I+A(k))$ has an eigenvalue zero, and we write $c_{\mathrm{r}}=R\left(k_{\mathrm{r}}\right) b\left(k_{\mathrm{r}}\right)$, so that $\left(I+A\left(k_{\mathrm{r}}\right)\right)^{-1} b\left(k_{\mathrm{r}}\right) \approx \frac{c_{\mathrm{r}}}{k-k_{\mathrm{r}}}$. Recalling that $c_{\mathrm{r}}$ is made up of the coefficients of the water and the plate, we write $c_{\mathrm{r}}=\left(c_{\mathrm{r} m}, \alpha_{\mathrm{r} n}\right)$.

5.2.1. Initial surface displacement. We discuss the case of an initial watersurface displacement. A similar expression for the case of a right-travelling pulse can be derived from expression (4.22) in the same way.

From (4.11), we obtain

$$
\left(\begin{array}{c}
Z(x, t) \\
W(z, t)
\end{array}\right)=\frac{2}{\sqrt{2 \pi}} \operatorname{Re}\left[\int_{0}^{\infty} \mathfrak{f}_{1}(k)\left(\begin{array}{c}
\hat{\zeta}(x, k) \\
\hat{w}(z, k)
\end{array}\right) \mathrm{e}^{-\mathrm{i} \omega(k) t} \mathrm{~d} k\right] .
$$

Since the contribution of the incident wave rapidly decreases for large times, we can approximate

$$
\left(\begin{array}{c}
Z(x, t) \\
W(z, t)
\end{array}\right) \sim \frac{2}{\sqrt{2 \pi}} \operatorname{Re}\left[\int_{0}^{\infty} \mathfrak{f}_{1}(k)\left(\begin{array}{c}
-\sum_{m=0}^{\infty} \hat{c}_{m}(k) \mathrm{e}^{k_{m}(k) x} \\
\sum_{n=0}^{\infty} \hat{\alpha}_{n} w_{n}(z)
\end{array}\right) \mathrm{e}^{-\mathrm{i} \omega(k) t} \mathrm{~d} k\right]
$$

for large times. We close the contour in the lower half plane, the additional contributions of which are negligible for large times (note that this contour is negatively oriented). Also using the approximation $\hat{c}_{m}(k) \approx \frac{c_{\mathrm{r} m}}{k-k_{\mathrm{r}}}$ and $\hat{\alpha}_{n}(k) \approx \frac{\alpha_{\mathrm{r} n}}{k-k_{\mathrm{r}}}$, the integral can be calculated using the residue theorem. This gives

$$
\left(\begin{array}{c}
Z(x, t) \\
W(z, t)
\end{array}\right) \sim-2 \sqrt{2 \pi} \operatorname{Re}\left[\mathrm{if}_{1}\left(k_{\mathrm{r}}\right)\left(\begin{array}{c}
-\sum_{m=0}^{\infty} c_{\mathrm{r} m} \mathrm{e}^{k_{m}\left(k_{\mathrm{r}}\right) x} \\
\sum_{n=0}^{\infty} \alpha_{\mathrm{r} n} w_{n}(z)
\end{array}\right) \mathrm{e}^{-\mathrm{i} \omega\left(k_{\mathrm{r}}\right) t}\right]
$$

for large $t$.

5.2.2. Initial plate displacement. For arbitrary initial plate displacement, we first note that, from (4.19),

$$
\mathfrak{f}(k) \sim \frac{\beta}{2 \sqrt{2 \pi}} \sum_{n=0}^{\infty} \alpha_{n}^{0} \lambda_{n}^{4} \frac{\bar{\alpha}_{\mathrm{r} n}}{k-\bar{k}_{\mathrm{r}}}
$$

near $k_{\mathrm{r}}$. Similarly,

$$
\left(\begin{array}{c}
\hat{\zeta}(x, k) \\
\hat{w}(z, k)
\end{array}\right) \sim \frac{1}{k-k_{\mathrm{r}}}\left(\begin{array}{c}
-\sum_{m=0}^{\infty} c_{\mathrm{r} m} \mathrm{e}^{k_{m}(k) x} \\
\sum_{n=0}^{\infty} \alpha_{\mathrm{r} n} w_{n}(z)
\end{array}\right),
$$

where we have neglected the contribution from the incident wave as before.

From (4.11), we have

$$
\left(\begin{array}{c}
Z(x, t) \\
W(z, t)
\end{array}\right)=\frac{2}{\sqrt{2 \pi}} \operatorname{Re}\left[\int_{0}^{\infty} \mathfrak{f}_{1}(k)\left(\begin{array}{c}
\hat{\zeta}(x, k) \\
\hat{w}(z, k)
\end{array}\right) \mathrm{e}^{-\mathrm{i} \omega(k) t} \mathrm{~d} k\right] .
$$


Closing the contour of integration in the forth quadrant and using the approximations (5.8) and (5.9) gives

$$
\left(\begin{array}{c}
Z(x, t) \\
W(z, t)
\end{array}\right) \sim-\mathbb{R e}\left[\frac{\mathrm{i} \beta}{k_{\mathrm{r}}-\bar{k}_{\mathrm{r}}} \sum_{j=0}^{\infty} \alpha_{j}^{0} \lambda_{j}^{4} \bar{\alpha}_{\mathrm{r} j}\left(\begin{array}{c}
-\sum_{m_{0}=0}^{\infty} c_{\mathrm{r} m} \mathrm{e}^{k_{m}\left(k_{\mathrm{r}}\right) x} \\
\sum_{n=0}^{\infty} \alpha_{\mathrm{r} n} w_{n}(z)
\end{array}\right) \mathrm{e}^{-\mathrm{i} \omega\left(k_{\mathrm{r}}\right) t}\right]
$$

for large $t$.

The formulas above can easily be extended to account for more than one complex scattering frequency near the real axis by also including the residues at these wavenumbers.

5.3. Accuracy of the approximation. The approximations derived in the previous subsection are for large times. A rough idea about how quickly this approximation becomes accurate can be obtained by looking at the double roots of the dispersion relation (3.4) as these double roots imply branch cuts of the integrand.

In the derivation of the approximate formulas (5.7) and (5.11), it is an important step to close the contour of integration in the forth quadrant of the complex plane so that it does not encircle any poles or branch cuts of the integrand other than the one specifically accounted for. The contribution from this extra integration path decays and this happens the quicker the further this path is away from the real axis (as the exponentials of the form $\mathrm{e}^{-\mathrm{i} \omega(k) t}$ decay faster for large $t$ if $\omega$ has larger negative imaginary part). The exact locations of the double roots are discussed in Appendix A.

Depending on the parameters of the problem, the roots of the dispersion relation (3.4) lie further away or closer to the real axis. The closer they lie, the longer it takes for the approximation to become accurate.

6. Numerical experiments. We present some results of numerical experiments. In all results shown, the plate stiffness and mass are chosen as $\beta=0.01$ and $\gamma=0.01$ and the water depth is $h=1$ (this can be thought of as having nondimensionalised with respect to water depth). All initial values not specifically mentioned in each subsection are assumed to vanish.

We begin with presenting results for the initial-boundary value problem with given water-surface displacement $(\S 6.1)$ and with plate displacement (§6.2) for fixed and free plate top. The validity of the approximation for large times is discussed thereafter $(\S 6.3)$.

6.1. Given water-surface displacement. For a plate extending above the water surface by a third of its length (i.e. $H=0.5$ ) and an initial water-surface displacement given by

$$
Z_{0}(x)=\frac{3}{4}(x+8) \mathrm{e}^{-(x+8)^{2} / 2},
$$

snapshots of the solution at $t=0,5,10,15,20,25,30,35,40$ are shown in figure 6.1 for a fixed plate and in figure 6.2 for a plate with freely moving top. The initial watersurface displacement results in two waves: one moving to the left and one moving to the right. The one travelling to the right eventually hits the elastic plate. Some energy is transferred to the plate, some is reflected as a wave travelling to the left. The energy transferred to the plate results in decaying oscillations of the plate, which in turn give rise to waves of decreasing amplitude travelling away from the plate. The qualitative behavior of the solution is similar for fixed and free top. 

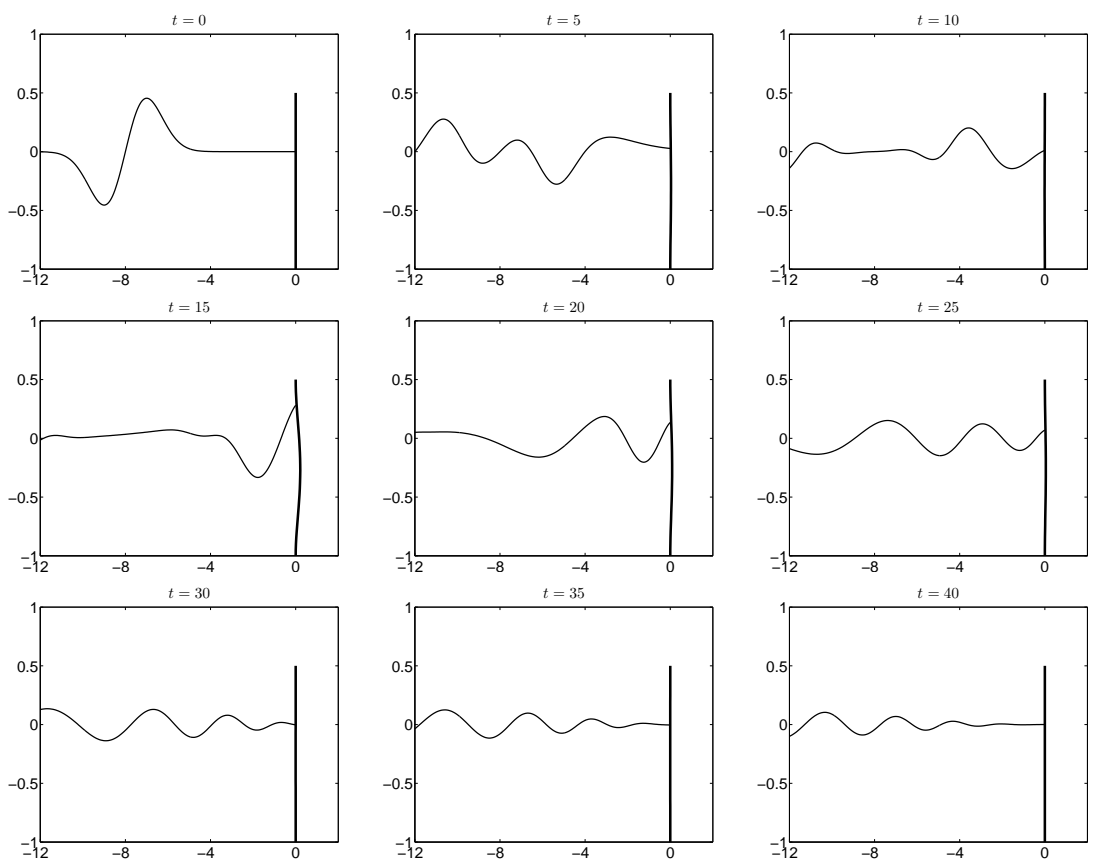

FIG. 6.1. Snapshots of the solution with fixed plate top for prescribed non-zero initial watersurface displacement.
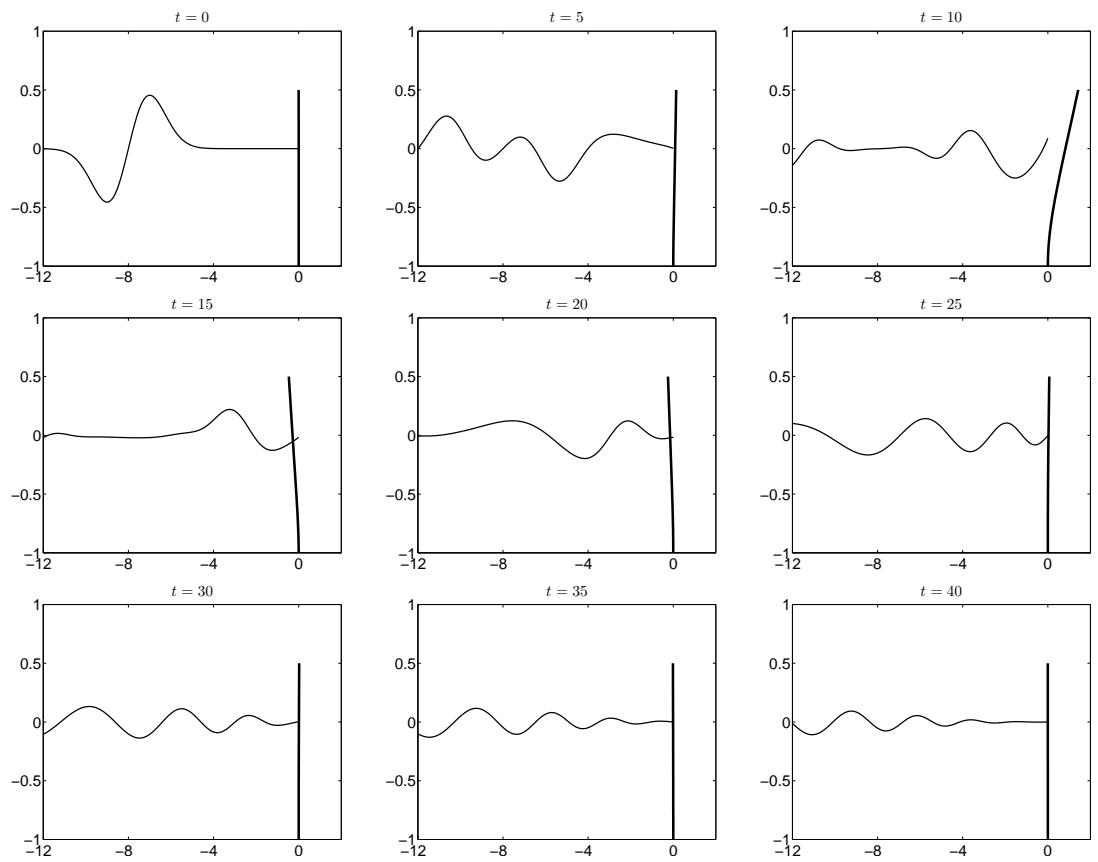

FIG. 6.2. Snapshots of the solution with free plate top for prescribed non-zero initial watersurface displacement. 

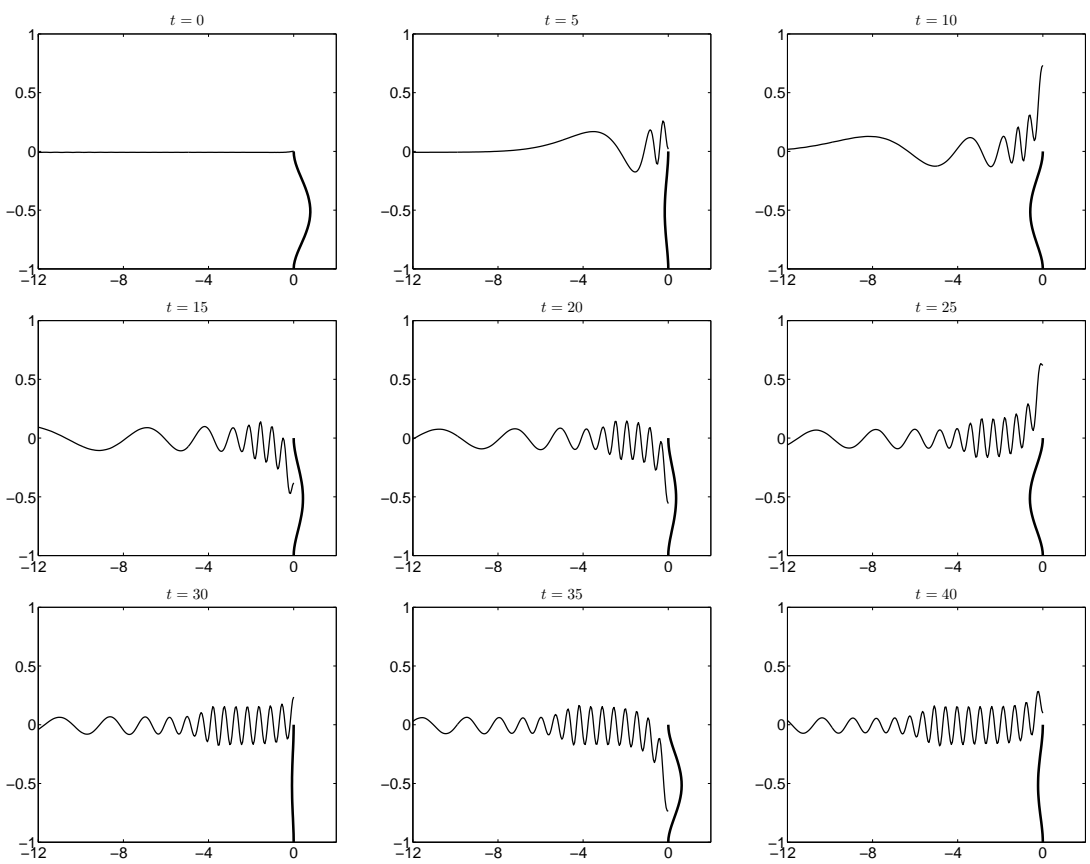

FIG. 6.3. Snapshots of the evolution of the solution for prescribed non-zero plate displacement in the first mode. The plate top is fixed and $H=0$.

6.2. Given plate displacement. For $H=0$ and fixed plate top, the evolution of an initial plate displacement in the first plate mode,

$$
W_{0}(z)=-0.5 w_{0}(z),
$$

is shown in figure 6.3. It can be seen that the plate, initially bent to the right, flexes to the left resulting in a long wave travelling away from the plate. Eventually, the plate keeps oscillating with slowly decreasing amplitude at a certain frequency, which results in a wave of that frequency travelling to the left.

This slowly decaying oscillatory behavior is connected with a complex scattering frequency near the real axis. This is investigated in more detail in §6.3. Similar results would have been obtained for $H=0.5$ but the effect is more pronounced for the shorter plate considered here $(H=0)$.

For $H=0.5$ and free plate top, snapshots of the evolution of an initial plate displacement in the second plate mode,

$$
W_{0}(z)=0.5 w_{1}(z)
$$

are shown in figure 6.4. Similarly to the previous case, there is an initial wave packet travelling away from the plate and an ongoing slowly decaying oscillation at a certain frequency.

If the plate is excited in the first mode (not shown), there are only very little ongoing oscillations.

6.3. Approximation for large times. The ongoing oscillations observed in the previous subsection are related to complex scattering frequencies near the real 

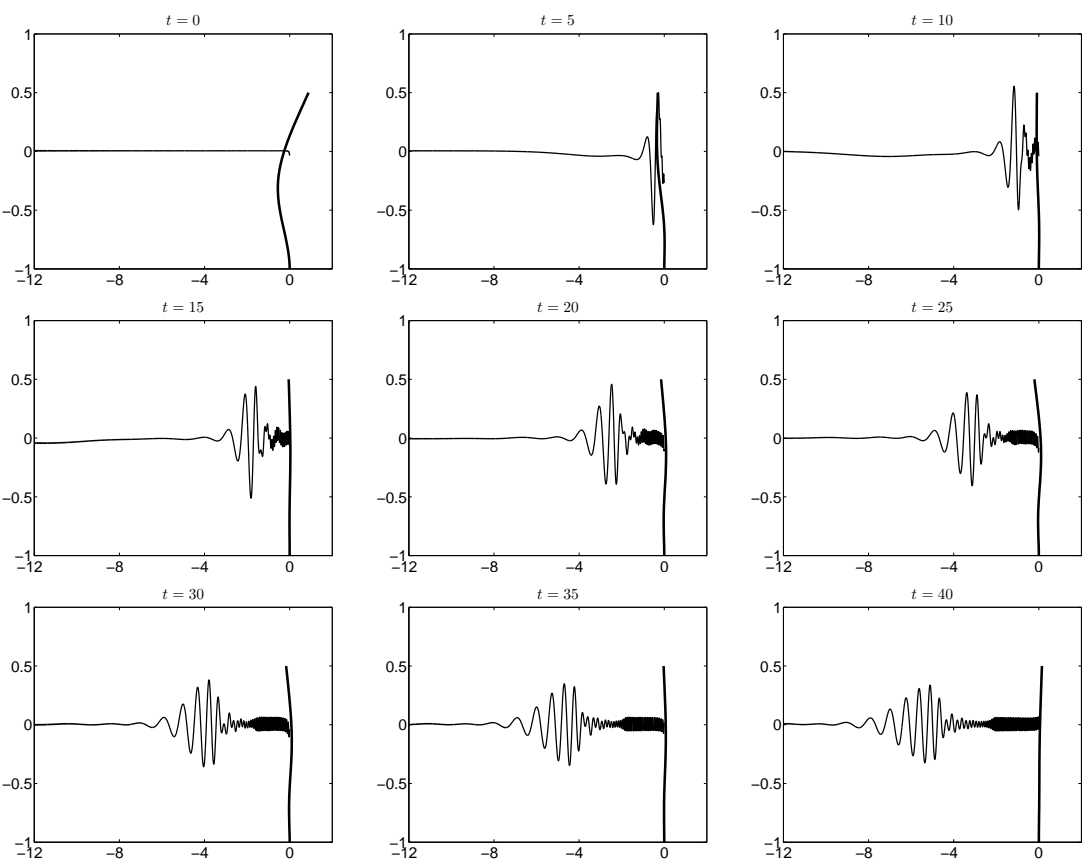

FIG. 6.4. Snapshots of the solution for prescribed non-zero plate displacement in the second mode. The plate top is free to move.
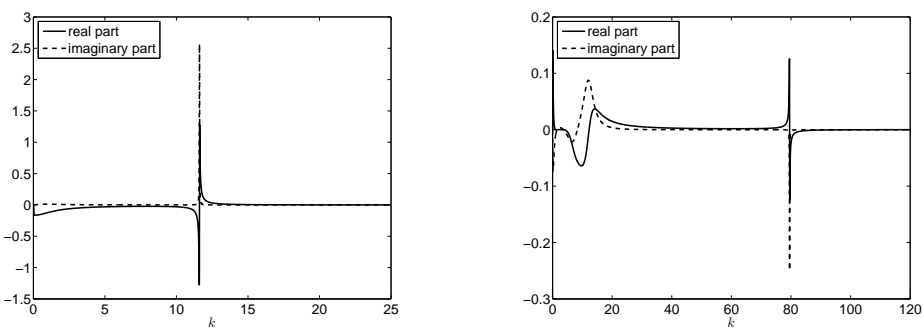

FIG. 6.5. Real and imaginary part of $\mathfrak{f}_{1}(k)$ for fixed plate (left; corresponding to fig. 6.3) and for free plate (right; corresponding to fig. 6.4).

axis. In particular, this can be observed as sharp spikes in the frequency-component function $\mathfrak{f}_{1}(k)$ (given by (4.19) in these cases), which is shown for both cases in figure 6.5. Using the method of [19], we find that there are complex scattering wavenumbers at $k_{\mathrm{r}} \approx 11.6116-0.0130 \mathrm{i}$ for the fixed plate (as in fig. 6.3 ) and $k_{\mathrm{r}} \approx 79.5379-0.0476 \mathrm{i}$ for the free plate (as in fig. 6.4), which precisely coincide with the location of the spikes in figure 6.5.

We want to use the approximation method of $\S 5.2$ to estimate the long-time behavior of the solution of the fixed plate initially bent in the first mode (as in fig. 6.3). Using the expression (5.11), the long-time behavior can be easily calculated. Figure 6.6 shows the behavior of the full solution (corresponding to figure 6.3) compared to the approximation obtained using (5.11). It can be seen that, after a short transient, the approximation quickly becomes accurate.

It is noteworthy that it is also possible to excite the mode corresponding to 

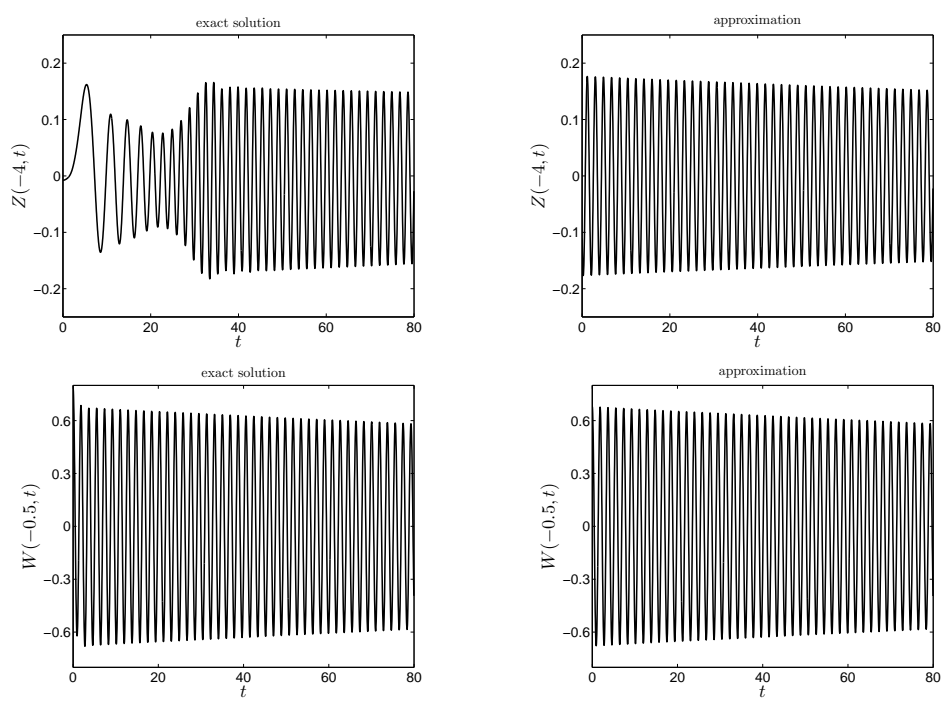

FIG. 6.6. Exact and approximate solution of the water-surface displacement at $x=-4$ and plate displacement at $z=-0.5$ versus time for fixed plate (corresponding to fig. 6.3).

the complex scattering frequency by an incident wave (rather than an initial plate displacement). Hitting the plate with a wave packet focused on $\operatorname{Re} k_{\mathrm{r}}$ results in an ongoing oscillation (not shown) similar to that resulting from the initial plate displacement.

7. Summary. A solution to the time-dependent problem of the interaction of a semi-infinite fluid domain with a free-surface bounded by a vertical elastic plate has been presented. The solution is constructed using a generalized-eigenfunction expansion and is valid for both initial fluid and initial plate displacements and velocities. This solution relies on a special inner product, in which the evolution operator is self-adjoint. We have then shown that an approximate solution can be found from this generalized-eigenfunction solution by contour integration and by finding the singularities of the solution extended analytically to complex frequencies.

Acknowledgements. This research was supported by Marsden grant UOO308 from the New Zealand government.

Appendix A. Some remarks on the dispersion relation for complex frequencies.

For real frequencies $\omega$, the parameter $\alpha=\omega^{2}$ in the dispersion relation (3.7),

$$
-k_{m} \tan k_{m} h=\alpha,
$$

is real and it is well-known that the equation has two purely imaginary roots $\pm k_{0}=$ $\mp \mathrm{i} k$ and a countable number of real roots $\pm k_{m}, m=1, \ldots, \infty$ [14]. The occurrence of the roots in pairs comes from the fact that $k \tan k h$ is an even function in $k$.

In the context of this work, it is important to understand the roots of the dispersion relation (A.1) for complex frequencies because extensive use is made of deforming the contour of integration, originally only containing real frequencies, into the forth quadrant of the complex plane. For the same reason, [5] investigate the solution of the dispersion relation for complex frequencies in detail. The same problem also arises 


$$
\begin{array}{cc}
z & \tilde{\alpha} \\
0+0 \mathrm{i} & 0+0 \mathrm{i} \\
1.1254+2.1062 \mathrm{i} & -1.6506-2.0600 \mathrm{i} \\
1.5516+5.3563 \mathrm{i} & -2.0578-5.3347 \mathrm{i} \\
1.7755+8.5367 \mathrm{i} & -2.2785-8.5226 \mathrm{i} \\
1.9294+11.6992 \mathrm{i} & -2.4311-11.6888 \mathrm{i}
\end{array}
$$

TABle A.1

First five values of $z$, for which (A.3) is satisfied, and corresponding values of $\tilde{\alpha}$, for which (A.2) has a double root.

in some water-wave problems involving porous structures and this is the reason why (A.1) involving complex right-hand sides is considered by [15]. We briefly summarize the results of these works in our notation as far as they are required here.

We first note that we consider only complex frequencies in the forth quadrant of the complex plane. Correspondingly, we discuss those roots of (A.1), which lie in the first quadrant (corresponding to $k_{m}, m=1, \ldots, \infty$ ) and in the third quadrant (corresponding to $k_{0}$ ).

One important property of (A.1) is that it may admit double roots for certain complex frequencies. The number of parameters can be reduced from two to only one by writing $z=k_{m} h$. Then, it is sufficient to look for solutions of

$$
-z \tan z=\tilde{\alpha}
$$

where $\tilde{\alpha}=\alpha h$. Equation (A.2) has a double root at $z$ precisely if

$$
\sinh 2 z+2 z=0
$$

is also satisfied. In this case, $z$ is the only double root of (A.2) (in the fourth quadrant). Finding the roots of (A.3) in the first quadrant and substituting into (A.2) gives the complex frequency parameter $\tilde{\alpha}$, for which (A.2) has a double root in the fourth quadrant. Again, it suffices to restrict to one quadrant of the complex plane. The first roots of (A.3) (if numbered with increasing real part) and the corresponding values of $\tilde{\alpha}$ are given in table $\mathrm{A}$.

\section{Appendix B. Eigenfunctions of the plate.}

In this section, the eigenfunctions of the plate, i.e. the eigenfunctions of the biharmonic operator subject to the fixed and free boundary conditions as introduced in $\S 2.2$, are briefly discussed.

The general form of the eigenfunctions of the bi-harmonic operator is given as

$$
\begin{aligned}
& w_{n}(z)=C_{1}\left(\cos \lambda_{n}(z+h)+\cosh \lambda_{n}(z+h)\right)+C_{2}\left(\cos \lambda_{n}(z+h)-\cosh \lambda_{n}(z+h)\right) \\
& +C_{3}\left(\sin \lambda_{n}(z+h)+\sinh \lambda_{n}(z+h)\right)+C_{4}\left(\sin \lambda_{n}(z+h)-\sinh \lambda_{n}(z+h)\right) .
\end{aligned}
$$

The coefficients $C_{j}$ and the eigenvalues $\lambda_{n}$ are determined by the boundary conditions as well as the restriction that $w_{n}$ has unit norm.

We always assume that we have a fixed bottom of the plate, i.e.

$$
w_{n}(-h)=0, \quad w_{n}^{\prime}(-h)=0,
$$

and consider the case of a fixed top or a free top at $z=H$. From the two boundary conditions (B.2), we find that $C_{1}=0$ and $C_{3}=0$, i.e.

$$
w_{n}(z)=C_{2}\left(\cos \lambda_{n}(z+h)-\cosh \lambda_{n}(z+h)\right)+C_{4}\left(\sin \lambda_{k}(z+h)-\sinh \lambda_{n}(z+h)\right) .
$$


B.1. Fixed plate top. If the plate is also fixed at $z=H$, we have

$$
w(H)=0, \quad w^{\prime}(H)=0
$$

in addition to (B.2). This gives

$$
\cos \lambda_{n}(H+h) \cosh \lambda_{n}(H+h)=1
$$

and allows us to express $C_{4}$ in terms of $C_{2}$. Substitution of the result into (B.3) gives

$$
\begin{aligned}
w_{n}(z) & =C_{2}\left(\left(\cos \lambda_{n}(z+h)-\cosh \lambda_{n}(z+h)\right)\right. \\
& \left.+\frac{\sin \lambda_{n}(H+h)+\sinh \lambda_{n}(H+h)}{\cos \lambda_{n}(H+h)-\cosh \lambda_{n}(H+h)}\left(\sin \lambda_{n}(z+h)-\sinh \lambda_{n}(z+h)\right)\right) .
\end{aligned}
$$

We choose $C_{2}$ such that $w_{n}$ has unit norm (this can be done analytically).

B.2. Free plate top. If the plate is free to move at $z=H$, we have

$$
w^{\prime \prime}(H)=0, \quad w^{\prime \prime \prime}(H)=0
$$

in addition to (B.2). This gives

$$
\cos \lambda_{n}(H+h) \cosh \lambda_{n}(H+h)=-1
$$

and

$$
\begin{aligned}
w_{n}(z) & =C_{2}\left(\left(\cos \lambda_{n}(z+h)-\cosh \lambda_{n}(z+h)\right)\right. \\
& \left.+\frac{\sin \lambda_{n}(H+h)-\sinh \lambda_{n}(H+h)}{\cos \lambda_{n}(H+h)+\cosh \lambda_{n}(H+h)}\left(\sin \lambda_{n}(z+h)-\sinh \lambda_{n}(z+h)\right)\right)
\end{aligned}
$$

for the free-plate-top case. Again, we choose $C_{2}$ such that $w_{k}$ has unit norm (this can be done analytically).

\section{Appendix C. Properties related to $\mathcal{A}$.}

C.1. Symmetry of $\mathcal{A}$. We want to show that the operator $\mathcal{A}$ defined in (4.3) is symmetric in the domain of $\mathcal{A}$ equipped with the inner product (4.4). We have

$$
\left\langle\left(\begin{array}{c}
Z \\
W
\end{array}\right), \mathcal{A}\left(\begin{array}{c}
Z^{\prime} \\
W^{\prime}
\end{array}\right)\right\rangle_{\mathcal{A}}=\left\langle Z, \mathcal{A}_{11} Z^{\prime}+\mathcal{A}_{12} W^{\prime}\right\rangle_{\Gamma^{\mathrm{f}}}+\beta\left\langle\partial_{z}^{2} W, \partial_{z}^{2}\left(\mathcal{A}_{21} Z^{\prime}+\mathcal{A}_{22} W^{\prime}\right)\right\rangle_{\Gamma^{\mathrm{p}}} .
$$

Consider each of the four summands separately. Using Green's second identity, we obtain

$$
\begin{aligned}
\left\langle Z, \mathcal{A}_{11} Z^{\prime}\right\rangle_{\Gamma^{\mathrm{f}}} & =\left\langle-\Psi,-\Psi_{n}^{\prime}\right\rangle_{\Gamma^{\mathrm{f}}}=\left\langle\Psi_{n}, \Psi^{\prime}\right\rangle_{\Gamma^{\mathrm{f}}}-\left\langle\Psi, \Psi_{n}^{\prime}\right\rangle_{\Gamma^{\mathrm{wp}}}+\left\langle\Psi_{n}, \Psi^{\prime}\right\rangle_{\Gamma^{\mathrm{wp}}} \\
& =\left\langle-\Psi_{n},-\Psi^{\prime}\right\rangle_{\Gamma^{\mathrm{f}}}-\left\langle-\gamma \Psi_{n}, \Psi_{n}^{\prime}\right\rangle_{\Gamma_{\mathrm{wp}}}+\left\langle\Psi_{n},-\gamma \Psi_{n}^{\prime}\right\rangle_{\Gamma^{\mathrm{wp}}} \\
& =\left\langle\mathcal{A}_{11} Z, Z^{\prime}\right\rangle_{\Gamma^{\mathrm{f}}}
\end{aligned}
$$

For the second summand, we can calculate

$$
\begin{aligned}
\left\langle Z, \mathcal{A}_{12} W^{\prime}\right\rangle_{\Gamma^{\mathrm{f}}} & =\left\langle-\Psi,-\Psi_{n}^{\prime}\right\rangle_{\Gamma_{\mathrm{f}}^{\mathrm{f}}}=-\left\langle\Psi, \Psi_{n}^{\prime}\right\rangle_{\Gamma^{\mathrm{pw}}}+\left\langle\Psi_{n}, \Psi^{\prime}\right\rangle_{\Gamma^{\mathrm{pw}}} \\
& =-\left\langle\Psi, \Psi_{n}^{\prime}\right\rangle_{\Gamma^{\mathrm{pw}}}-\left\langle\Psi_{n}, \gamma \Psi_{n}^{\prime}\right\rangle_{\Gamma^{\mathrm{pw}}}-\left\langle\Psi_{n}, \beta \partial_{z}^{4} W^{\prime}\right\rangle_{\Gamma^{\mathrm{pw}}} \\
& =-\left\langle\Psi, \Psi_{n}^{\prime}\right\rangle_{\Gamma^{\mathrm{pw}}}-\left\langle\Psi_{n}, \gamma \Psi_{n}^{\prime}\right\rangle_{\Gamma^{\mathrm{pw}}}-\beta\left\langle\chi \Psi_{n}, \partial_{z}^{4} W^{\prime}\right\rangle_{\Gamma^{\mathrm{p}}} \\
& =-\left\langle\Psi, \Psi_{n}^{\prime}\right\rangle_{\Gamma^{\mathrm{pw}}}-\left\langle\Psi_{n}, \gamma \Psi_{n}^{\prime}\right\rangle_{\Gamma^{\mathrm{pw}}}-\beta\left\langle\partial_{z}^{2} \partial_{t}^{2} W, \partial_{z}^{2} W^{\prime}\right\rangle_{\Gamma^{\mathrm{p}}} \\
& =-\left\langle\Psi, \Psi_{n}^{\prime}\right\rangle_{\Gamma^{\mathrm{pw}}}-\left\langle\Psi_{n}, \gamma \Psi_{n}^{\prime}\right\rangle_{\Gamma^{\mathrm{pw}}}+\beta\left\langle\partial_{z}^{2} \mathcal{A}_{21} Z, \partial_{z}^{2} Z^{\prime}\right\rangle_{\Gamma^{\mathrm{p}}}
\end{aligned}
$$


Similarly, the third term equates to

$$
\begin{aligned}
\beta\left\langle\partial_{z}^{2} W, \partial_{z}^{2} \mathcal{A}_{21} Z^{\prime}\right\rangle_{\Gamma^{\mathrm{p}}} & =-\beta\left\langle\partial_{z}^{2} W, \partial_{z}^{2} \partial_{t}^{2} W^{\prime}\right\rangle_{\Gamma^{\mathrm{p}}}=-\beta\left\langle\partial_{z}^{4} W, \partial_{t}^{2} W^{\prime}\right\rangle_{\Gamma^{\mathrm{p}}} \\
& =\beta\left\langle\partial_{z}^{4} W, 1 / \gamma \chi \Psi^{\prime}\right\rangle_{\Gamma^{\mathrm{p}}}=-\beta\left\langle\partial_{z}^{4} W, \Psi_{n}^{\prime}\right\rangle_{\Gamma^{\mathrm{pw}}} \\
& =\left\langle-\gamma \Psi_{n}-\beta \partial_{z}^{4} W, \Psi_{n}^{\prime}\right\rangle_{\Gamma^{\mathrm{pw}}}+\left\langle\gamma \Psi_{n}, \Psi_{n}^{\prime}\right\rangle_{\Gamma^{\mathrm{pw}}} \\
& =\left\langle\Psi, \Psi_{n}^{\prime}\right\rangle_{\Gamma^{\mathrm{pw}}}+\left\langle\gamma \Psi_{n}, \Psi_{n}^{\prime}\right\rangle_{\Gamma^{\mathrm{pw}}} \\
& =\left\langle-\Psi_{n},-\Psi^{\prime}\right\rangle_{\Gamma^{\mathrm{f}}}+\left\langle\Psi_{n}, \Psi^{\prime}\right\rangle_{\Gamma^{\mathrm{pw}}}+\left\langle\gamma \Psi_{n}, \Psi_{n}^{\prime}\right\rangle_{\Gamma^{\mathrm{pw}}} \\
& =\left\langle\mathcal{A}_{12} W, Z^{\prime}\right\rangle_{\Gamma^{\mathrm{f}}}+\left\langle\Psi_{n}, \Psi^{\prime}\right\rangle_{\Gamma^{\mathrm{pw}}}+\left\langle\gamma \Psi_{n}, \Psi_{n}^{\prime}\right\rangle_{\Gamma^{\mathrm{pw}}}
\end{aligned}
$$

The calculation for the last term is

$$
\begin{aligned}
& \beta\left\langle\partial_{z}^{2} w, \partial_{z}^{2} \mathcal{A}_{22} w^{\prime}\right\rangle_{\Gamma^{\mathrm{p}}}=-\beta\left\langle\partial_{z}^{2} W, \partial_{z}^{2} \partial_{t}^{2} W^{\prime}\right\rangle_{\Gamma^{\mathrm{p}}}=-\beta\left\langle\partial_{z}^{4} W, \partial_{t}^{2} W^{\prime}\right\rangle_{\Gamma^{\mathrm{p}}} \\
& \quad=\left\langle\gamma \partial_{t}^{2} W+\chi \Psi,-\beta / \gamma \partial_{z}^{4} W^{\prime}-1 / \gamma \chi \Psi^{\prime}\right\rangle_{\Gamma^{\mathrm{p}}} \\
& \quad=-\beta\left\langle\partial_{t}^{2} W, \partial_{z}^{4} W^{\prime}\right\rangle_{\Gamma^{\mathrm{p}}}-\left\langle\chi \Psi, \beta / \gamma \partial_{z}^{4} W^{\prime}\right\rangle_{\Gamma^{\mathrm{p}}}-\left\langle\chi \Psi, 1 / \gamma \chi \Psi^{\prime}\right\rangle_{\Gamma^{\mathrm{p}}}-\left\langle\partial_{t}^{2} W, \chi \Psi^{\prime}\right\rangle_{\Gamma^{\mathrm{p}}} \\
& \quad=-\beta\left\langle\partial_{z}^{2} \partial_{t}^{2} W, \partial_{z}^{2} W^{\prime}\right\rangle_{\Gamma^{\mathrm{p}}}+\left\langle\Psi, 1 / \gamma\left(\Psi^{\prime}+\gamma \Psi_{n}^{\prime}\right)\right\rangle_{\Gamma^{\mathrm{pw}}}-\left\langle\Psi, 1 / \gamma \Psi^{\prime}\right\rangle_{\Gamma^{\mathrm{pw}}}-\left\langle\Psi_{n}, \Psi^{\prime}\right\rangle_{\Gamma^{\mathrm{pw}}} \\
& \quad=\beta\left\langle\partial_{z}^{2} \mathcal{A}_{22} W, \partial_{z}^{2} W^{\prime}\right\rangle_{\Gamma^{\mathrm{p}}}+\left\langle\Psi, \Psi_{n}^{\prime}\right\rangle_{\Gamma^{\mathrm{pw}}}-\left\langle\Psi_{n}, \Psi^{\prime}\right\rangle_{\Gamma^{\mathrm{pw}}} \\
& =\beta\left\langle\partial_{z}^{2} \mathcal{A}_{22} W, \partial_{z}^{2} W^{\prime}\right\rangle_{\Gamma^{\mathrm{p}}}
\end{aligned}
$$

where the last two terms add up to zero by Green's second identity (since $\Psi=0$ on $\Gamma^{f}$ ) and we have used the edge conditions for the plate twice.

Adding the last four equations leaves

$$
\left\langle\left(\begin{array}{c}
Z \\
W
\end{array}\right), \mathcal{A}\left(\begin{array}{c}
Z^{\prime} \\
W^{\prime}
\end{array}\right)\right\rangle_{\mathcal{A}}=\left\langle\mathcal{A}\left(\begin{array}{c}
Z \\
W
\end{array}\right),\left(\begin{array}{c}
Z^{\prime} \\
W^{\prime}
\end{array}\right)\right\rangle_{\mathcal{A}},
$$

where $-\left\langle\Psi, \Psi_{n}^{\prime}\right\rangle_{\Gamma^{\mathrm{pw}}}+\left\langle\Psi_{n}, \Psi^{\prime}\right\rangle_{\Gamma^{\mathrm{pw}}}=0$ follows from Green's second identity since $\Psi=0$ on $\Gamma^{\mathrm{f}}$.

C.2. Normalization. The normalization of the eigenfunctions is achieved by using the result that the eigenfunctions satisfy the same normalizing condition with and without the scattering terms. This result, the proof of which is quite technical, is well-known and has been shown for many different situations. The original proof was for Schrödinger's equation and was due to $[22,10]$. A proof for the case of the Helmholtz equation was given by [27]. Recently the proof was given for water waves by $[6,7]$. In none of these papers were there any calculations made, nor was the domain semi-infinite.

We assume that the eigenfunctions satisfy the same normalizing condition with and without the scattering terms. Note that no scattering means that the plate does not move and hence, the incident wave is fully reflected, i.e. $\hat{\alpha}_{m}=0$ and $\hat{c}_{m}=\delta_{0 m}$ for all $m \in \mathbb{N}$. Thus, noting that $k_{0}=-\mathrm{i} k$ and $\kappa_{0}=-\mathrm{i} \kappa$, we obtain

$$
\begin{aligned}
\left\langle\left(\begin{array}{c}
\hat{\zeta}(\cdot, k) \\
\hat{w}(\cdot, k)
\end{array}\right),\left(\begin{array}{c}
\hat{\zeta}(\cdot, \kappa) \\
\hat{w}(\cdot, \kappa)
\end{array}\right)\right\rangle_{\mathcal{A}} & =\int_{\Gamma^{\mathrm{f}}}\left(\mathrm{e}^{-k_{0} x}+\mathrm{e}^{k_{0} x}\right) \overline{\left(\mathrm{e}^{-\kappa_{0} x}+\mathrm{e}^{\kappa_{0} x}\right)} \mathrm{d} x \\
& =4 \int_{-\infty}^{0} \cos k x \cos \kappa x \mathrm{~d} x=2 \pi \delta(k-\kappa) .
\end{aligned}
$$




\section{REFERENCES}

[1] J. T. BEALE, Eigenfunction expansions for objects floating in an open sea, Comm. Pure Appl. Math., 30 (1977), pp. 283-313.

[2] Task Committe, ed., Wave Forces on Inclined and Vertical Wall Structures, ASCE, 1995.

[3] R. Dautray and J. L. Lions, Mathematical analysis and numerical methods for science and technology, vol. 5, Springer, 1992.

[4] A. Friedman and M. Shinbrot, The initial value problem for the linearized equations of water waves, J. Math. \& Mech., 17 (1967), pp. 107-180.

[5] C. Hazard and M. Lenoir, Determination of scattering frequencies for an elastic floating body, SIAM J. Math. Anal., 24 (1993), pp. 1458-1514.

[6] — Surface water waves, in Scattering, R. Pike \& P. Sabatier, ed., Academic, 2002, pp. 618636.

[7] C. Hazard and F. Loret, Generalized eigenfunction expansions for scattering problems with an application to water waves, Proc. Roy. Soc. Edinburgh, 137A (2007), pp. 995-1035.

[8] — The singularity expansion method applied to the transient motions of a floating elastic plate, Math. Mod. Num. Anal., 41 (2007), pp. 925-943.

[9] C. Hazard and M. H Meylan, Spectral theory for a two-dimensional elastic thin plate floating on water of finite depth, SIAM J. Appl. Math., 68 (2007), pp. 629-647.

[10] T. IKeBE, Eigenfunction expansions associated with the Schroedinger operators and their applications to scattering theory, Arch. Rational Mech. Anal., 5 (1960), pp. 1-34.

[11] M. S. Kirkgoz, A. K. Tanrikulu, and C. Dündar, Dynamic analysis of a vertical plate exposed to breaking wave impact, Ocean Engng, 31 (2004), pp. 1623-1635.

[12] A. Korobkin and S. Malenica, Steep wave impact onto elastic wall, in Proc. 22nd Int. Workshop on Water Waves and Floating Bodies, Plitvice, Croatia, S. Malenica and I. Senjanovic, eds., 2007, pp. 33-36.

[13] P. D. Lax and R. S. Phillips, Scattering Theory, Academic, 1989.

[14] C. M. Linton and P. McIver, Handbook of Mathematical Techniques for Wave / Structure Interactions, Chapman \& Hall /CRC, Boca Raton, Florida, 2001. 304 pp.

[15] P. McIver, The dispersion relation and eigenfunction expansions for water waves in a porous structure, J. Engng Math., 34 (1998), pp. 319-334.

[16] C. C. MeI, The Applied Dynamics of Ocean Surface Waves, World Scientific, 1989.

[17] M. H. MeYlan, Generalized eigenfunction expansion for linear water waves, J. Fluid Mech., (2009). in press.

[18] M. H. Meylan and R. Eatock TAYlor, Time-dependent water-wave scattering by arrays of cylinders and the approximation of near trapping, J. Fluid Mech., (2009). in press.

[19] M. H. Meylan and L. Gross, A parallel algorithm to find the zeros of a complex analytic function, ANZIAM J., 44 (2003), pp. E216-E234.

[20] D. H. Peregrine, Water-wave impact on walls, Annu. Rev. Fluid Mech., 35 (2003), pp. 23-43.

[21] M. A. Peter and M. H. Meylan, Time-dependent interaction of water waves and a vertical elastic plate, in Proc. 23rd Int. Workshop on Water Waves and Floating Bodies, Jeju, Korea, H. S. Choi and Y. Kim, eds., 2008, pp. 144-147.

[22] A. YA. Povzner, On the expansions of arbitrary functions in terms of the eigenfunctions of the operator $-\Delta u+c u$ (in Russian), Mat. Sbornik, 32 (1953), pp. 109-156.

[23] V. A. Squire, Of ocean waves and sea-ice revisited, Cold Regions Science and Technology, 49 (2007), pp. 110-133.

[24] V. A. Squire, J. P. Dugan, P. Wadhams, P. J. Rottier, and A. J. Liu, Of ocean waves and sea ice, Annu. Rev. Fluid Mech., 27 (1995), pp. 115-168.

[25] S. Steinberg, Meromorphic families of compact operators, Arch. Rational Mech. Anal., 31 (1968), pp. 372-379.

[26] E. Watanabe, T. Utsunomiya, and C.M. Wang, Hydroelastic analysis of pontoon-type vlfs: a literature survey, Eng. Struct., 26 (2004), pp. 245-256.

[27] C. H. Wilcox, Scattering Theory for the d'Alembert Equation in Exterior Domains, SpringerVerlag, 1975. 